with crashes involving older drivers (OD; 20 years+) was carried out to help target prevention efforts. STATS19 data were analysed for 2000-2007 for Wales. Crashes involving YD and OD were identified. Casualties were grouped into pedestrians and passengers and analysed by age, sex and severity of injury. There were 12191 pedestrian casualties and 32265 passenger casualties. Pedestrians injured in YD crashes ( $n=784,6.4 \%$ ) were more likely to die or suffer killed and serious injury (KSI) than those in OD crashes $(25.5 \%$ vs $18.8 \%$; OR 1.48, 95\% CI 1.25 to 1.75). Passengers in YD vehicles in YD crashes ( $n=5629,9.0 \%$ ) were also more likely to be KSI $(12.0 \%)$, than those in OD vehicles in YD crashes $(6.6 \%$; OR $1.92,95 \%$ CI 1.59 to 2.33$)$ or $O D$ vehicles in $\mathrm{OD}$ crashes (8.7\%; OR 1.44, 95\%CI 1.30 to 1.59). Crashes involving YD cause significantly more serious and fatal injuries to pedestrians and passengers travelling with the YD. Interventions to address these crashes are required urgently. Many countries have successfully used Graduated Driver Licensing to reduce new YD crash risk. It is time that such an approach was implemented in the UK.

\section{ROAD TRAFFIC CRASH CASUALTY INJURY SEVERITY CRASHES INVOLVING YOUNG DRIVERS COMPARED WITH OLDER DRIVERS}

S J Jones*, S R Palmer Correspondence: Department of Primary Care and Public Health , Cardiff University / Public Health Wales, Cardiff University, 5th Floor, Neuadd Meirionnydd, Heath Park, Cardiff CF14 4XN, UK

10.1136/ip.2010.029215.531

In the UK, road traffic crashes are declining for all except young drivers (YD; aged 17-19). Comparison of the severity of injuries to passengers and pedestrians in YD crashes 